\title{
ANALYSE D'UNE PRODUCTION ÉCRITE ET ORALE : SENS EN CONTEXTE ÉNONCIATIF DU FRANÇAIS LANGUE ÉTRANGÈRE
}

Rudy Kohwer ${ }^{*}$

RÉSUMÉ: Dire aujourd'hui que la faute de syntaxe et la confusion lexicale soient inexistantes dans les énoncés écrits et parlés d'apprenants du Français Langue Seconde (FLS), est erroné. Ce n'est donc pas les apports théoriques de la linguistique structurale et de la psychologie béhavioriste des années 1950 qui corrigent selon une pédagogie d'enseignement pourtant déjà fondée sur l'abordage par compétences de communication. Si nous partageons par experience certains manques dans la méthode de français Latitudes 1 (2008) à appliquer par le manuel du professeur, c'est que tout s'oppose à enseigner le mode de combinaison ensemble avec l'interprétation des objets pour l'apprentissage des structures syntaxiques des énoncés et leur interprétation à l'orale. Et cela malgré la fréquence d'activités de répétition d'expressions simples en contexte déterminé par les trois actes de language inviter et répondre à une invitation, demander et indiquer l'beure et, prendre et fixer un rendez-vous, lesquels contextualisent une tâche finale soumise à analyse. Afin de mieux appréhender les règles pour des structures syntaxiques et des représentations de signifié bien formées dans les énoncés d'apprenants, il nous paraît pertinent de développer par l'approche analytique et contemporaine de la philosophie du langage. Après une partie consacrée à la présentation du corpus de référence et en rendant compte de la complexité du problème de recherche, nous analyserons une production langagière d'apprenante par les outils desdites approches imposés sur l'objet d'étude suivant : les descriptions syntaxiques et les représentations de signifié.

MOTS-CLÉS: Monde; Sens; Signifié; Syntaxe; Verbe.

\section{Valeurs sémantiques du verbe: Jusqu'à la philosophie analytique et contemporaine du langage}

Revenons sur les apports théoriques de la psychologie béhavioriste et de la linguistique structurale considérés par la tradition méthodologique audio-visuelle et structurale. Si ce premier courant prétend renforcer le contexte socioculturel et ce deuxième exclure l'erreur du contexte énonciatif, nous nous opposons. Les jeux de répétition sur les contenus des activités de fixation conditionnent des réseaux d’habitude et nous ajoutons, par

* Doutorando em Memória: Linguagem e sociedade pela Universidade Estadual do Sudoeste da Bahia (Uesb). Mestre em Linguística Aplicada pela Universidade Federal da Grande Dourados (UFGD). Bolsista Capes. 
conditionnement d'expressions simples et non complexes afin de confirmer notre opposition. Référents du discours pédagogique et didactique du Cadre européen de référence pour les langues (CECRL), ces deux apports fondèrent la méthode d'acquisition des compétences de communication avant l'intégration de la perspective actionnelle.

Cependant et en terme béhavioriste de comportement, ce paradigme de la psychologie scientifique conditionnait déjà une méthode pragmatique et par l'histoire des interactions de l'individu avec son environnement. Mais ce que nous pouvons lui reprocher est la répétition des mêmes réseaux d'habitudes dans les mondes, ainsi, son utilisation pragmatique du signe. En effet, nous croyons difficile de répéter pour mémoriser les expressions complexes bien comme le contexte énonciatif dans lequel elles sont prises et qui renforce leur signification par le sens du tout. Et quant à la description d'un objet, elle se fait prise dans la langue comme système linguistique isolé. Donc, le contexte socioculturel sous l'objet ne peut être considéré, ainsi, ni même renforcé lorsque pour cela la langue doit être prise dans sa dimension externe, subjective, en somme, ce que représente le contenu propositionnel d'une expression.

La perspective actionnelle comme acquisition de compétences de communication, est la condition première de production et un principe du CECRL depuis le début de ce siècle. Celui-ci vise l'autonomisation de l'apprenant s'engageant alors individuellement pour une action dans un cadre social donné. Quatre composantes - linguistique, discursive, référentielle, socioculturelle - comme substrat de cette compétence de communication, interrogent Moirand (1982) sur leur fonctionnement conjoint pour une stratégie d'apprentissage corrigeant l'erreur. Si Bérard (1991) l'introduit dans son article L'approche communicative. Théorie et pratiques, signalons que le problème - la présence de représentations de signifié de la langue maternelle dans les énoncés en langue étrangère - est la caractéristique de cette erreur. En somme, nous développons pourquoi sa présence dans les contextes énonciatifs d'apprenants et cela malgré cette nouvelle perspective. Nous avons constaté une divergence, à savoir, si pour Moirand (1982) une telle stratégie intervient seulement lors des activités d'acquisition de ladite compétence, pour Canale et Swain (1980) elle est à enseigner dès le début de l'apprentissage. 
La première direction est la correction relevant de «stratégies individuelles de communication" (MOIRAND, 1982, p. 20), alors que la deuxième orientation s'oppose par des stratégies collectives lorsqu'enseignées initialement selon Canale et Swain (1980). En effet, une experience de professeur confirme que, les premiers apprentissages à l'acquisition des connaissances socioculturelles initiales et des premières constructions syntaxiques, sont communiqués de forme collective, ou mieux, par des interactions impliquant le groupe classe dans son ensemble. En somme et selon les conclusions de Bérard (1991, p. 20) sur cette réflexion problématisant l'imposition d'une compétence stratégique pour corriger, s'il convient de s'interroger «sur le rapport social/individuel», nous avons développé les motifs intégrant la perspective actionnelle, c'est-à-dire l'action sociale depuis les années 2000 dans le CECRL. Or, si cette dernière engage individuellement l'apprenant dans son action sociale, ainsi, tendant à démontrer l'intérêt pour la composante référentielle - la connaissance des domaines d'expérience et des objets du monde et de leur relation - proposée par Moirand (1982) en réponse à Canale et Swain (1980), la relation entre ces quatre composantes parait alors obsolète, l'erreur est encore présente, et la perspective chomskienne ressort lorsque la compétence linguistique, c'est-à-dire et ici l'individuel, est séparée de la performance, c'est-à-dire le social.

En revanche, nous croyons sans réserve l'idée chomskienne cité par Bérard (1991, p. 13) : Sa position «met en doute l'efficacité des théories de référence dans le cadre de l'enseignement des langues ». Et pour cause, quelques années plus tard Chomsky (2009, p. 187, traduction libre) établit des résultats sur l'étude des descriptions structurelles soutenue par l'explication comme mode de raisonnement scientifique vers l'hypothèse des structures profondes qu’il applique au courant de la linguistique générative : «Les règles qui relationnent les structures syntaxiques avec les représentations de signifié ne sont d'aucune manière bien comprises ».

Si l'on poursuit sur l'apport de nouvelles connaissances par cette linguistique à propos des systèmes de règles, car capable d'expliquer certaines relations de descriptions structurelles expérimentables par les transformations grammaticales, on doit cependant considérer, pour notre objet d'étude, d'autres descriptions liées aux valeurs sémantiques du 
verbe. Elles sont celles ne relevant pas des principes de la grammaire universelle : Les autres manières dont s'interprètent, se forment et s'utilisent les énoncés. De manière générale, nous pensons que le système pragmatique est aussi à considérer comme hypothèse du tout, à savoir, signification, communication, action et intention en relation dans le contexte énonciatif, contribueraient aux règles qui relationnent les structures syntaxiques avec les représentations de signifié.

L'examen de ce tout à partir d'un énoncé d'apprenante montre aussi bien que la signification de l'objet être dans Je serais plus contente que tu m'accompagnes et la construction syntaxique du verbe accompagner sont incomprises. Nous questionnons déjà l'erreur : Faitelle défaut au sens et à la signification du contexte énonciatif bien formé en langue étrangère ? Nous allons donc tenter de le vérifier à partir du corpus de référence où l'erreur se trouve et prélevé dans un contexte pédagogique concret, à savoir, la salle de classe d'une école particulière de langues où une apprenante a réalisé la tâche finale comme perspective actionnelle dans la méthode de français Latitudes 1 des auteurs Régine Mérieux et Yves Loiseau (2008, p. 63). La consigne de la tâche finale est la suivante : «Vous téléphonez à un ami pour lui proposer d'aller au cinéma. Vous parlez des films, des heures, puis vous fixez un rendez-vous à votre ami. Regardez le programme et, par deux, imaginez puis jouez un dialogue. Écrivez un courriel à un autre ami pour l'inviter à venir avec vous ».

Lors de l'écriture du courriel, l'apprenante a rédigé l'erreur Je vais rester plus content de votre companie. Agrammaticale en français quant à la représentation du signifié rester, l'apprenante montre alors un raisonnement par sa langue maternelle. Cela parce que l' expression ficar est bien formée en portugais du Brésil, et surtout à partir de laquelle elle traduit littéralement lexique et syntaxe. Un tel problème reposant sur un contenu propositionnel lié à la forme de l'énoncé, est réalisé pour une production écrite puis interprété à l'orale selon la sollicitation du professeur et devant le groupe classe. Ci-dessous, le corpus de référence composé du contexte énonciatif écrit : 
Courriel 1 - Corpus de référence

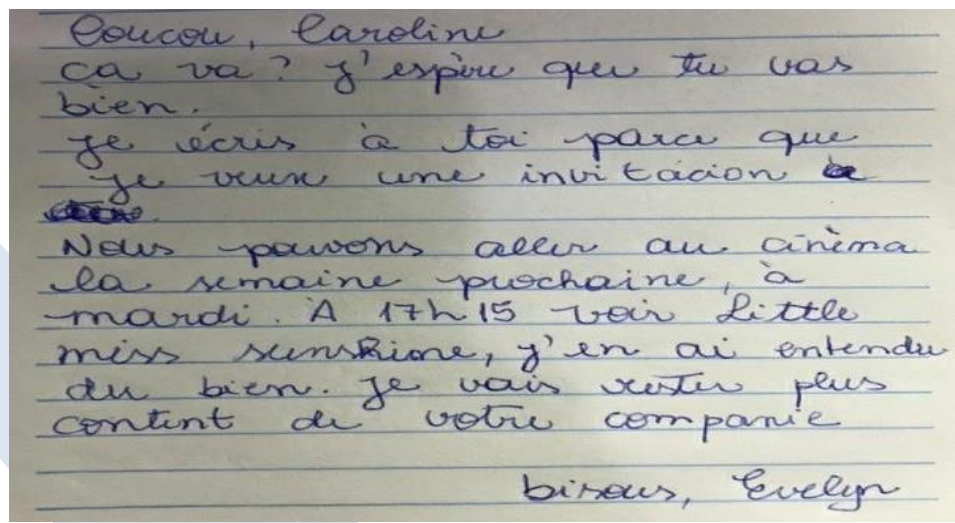

Source: Contenu élaboré par l'étudiante (2020)

Nous reformulons et étudions le problème: La réalisation des représentations de signifié de la langue maternelle dans les énoncés en langue étrangère. Nous allons en ce sens au delà du principe explicatif quant à la linguistique générative et des théories distributionnelles quant aux grammaires structurelles. Roulet (1972, p. 199) produit deux critiques dans son article Théories grammaticales et pédagogie des langues : 1) Sur les grammaires structurelles à propos du traitement de deux aspects du verbe. En sommes, elles ne réalisent pas « l'inventaire des constructions syntaxiques (compléments nominaux ou propositionnels) dans lesquels les verbes peuvent entrer et la description des valeurs sémantiques (temporelles, aspectuelles, etc) que les formes étudiées peuvent prendre dans le discours ». Et, 2) sur la grammaire générative chomskienne. Restant une grammaire de la phrase et du système de la langue, elle « ne peut fournir par conséquent qu’un apport limité à une pédagogie qui ne vise pas seulement la maîtrise du système grammatical mais celle de l'emploi de la langue comme instrument de communication » (p. 201).

Avec ce type d'erreur, rester appartient à une signification de la langue maternelle. Se faisant, nous constatons que la langue seconde est étrangère dans le sens d'étrangère à l'apprenante. Si nous rappelons la traduction littérale de serais par rester, alors nous montrons deux représentations de signifié distinctes. Ainsi, force est de constater deux significations, 
respectivement, être plus contente limité au moment de la compagnie et rester plus contente sans limite de temps. Mais pourtant, ficar traduit littéralement par rester représente dans la langue maternelle ce que représente serais dans la langue d'apprentissage, lequel doit alors se concentrer sur ce que représentent être et rester dans la langue seconde, confusion ressortant fréquemment dans les productions écrites et orales d'apprenants.

\section{Analyse du sens en contexte énonciatif: Être ou rester ?}

Dans cette première partie pour l'examen de l'erreur, nos motivations furent le raisonnement de l'apprenante. Sa langue maternelle a croisé notre regard sur la langue seconde afin d'opposer la tradition pédagogique et lesdites grammaires scientifiques à la résolution du cas en observation. Dans une analyse des significations sur rester et sur être, nous déplacerons le problème l'imposant à l'outils indiqué par Davidson (1967) cité dans la méthode Introduction à la logique. La logique classique des propositions et des prédicats de Keller (2007, p. 19) : Le principe de compositionnalité « nous permettait d'apprendre une langue et de connaitre les significations de phrases entièrement nouvelles (encore jamais entendues) sur la base de notre connaissance des mots qu'elles contiennent et des règles grammaticales ».

Mais lorsque nous tenons compte aussi de la communication et de l'intention dans l'énoncé et à un niveau contextuel, nous nous devons d'ajouter à l'examen de la signification par la logique moderne de Frege (1879) centrée sur une méthode logico-extensionnelle en philosophie analytique, l'hypothèse de la forme logique étudié par Vanderveken (1992) et par Searle (1969) en théorie des actes de discours du courant de la philosophie contemporaine du langage : Si la proposition subordonnée complétive que tu m'accompagnes renvoi à l'action future d'être accompagnée et si la proposition principale Je serais plus contente accomplie l'intention de l'apprenante d'une volonté, alors action et intention produiront son comportement, en somme, lorsque son sentiment d'être plus contente sera accomplie par l'action d'être accompagnée au cinéma. Est-ce que le comportement est modifié par l'accomplissement de la volonté de l'apprenante dans le contexte énonciatif ? Nous cherchons à le vérifier.

Nous constatons que le contexte énonciatif, premièrement, ne réalise pas l'action, c'est-à-dire le monde où se vérifie le comportement de l'apprenante pendant la sortie au 
cinéma. Nous pouvons dire alors que l'action n'est pas satisfaite selon l'hypothèse de la forme logique et Vanderveken (1992, p. 14) l'entend lorsque « le monde ne correspond pas à son contenu propositionnel », ici, Je serais plus contente que tu m'accompagnes. En revanche et deuxièmement, le contexte énonciatif accomplit l'intention de l'apprenante, car en disant Je vais rester plus content de ta companie, l'apprenante le fait par Je écris à toi parce que je veux une invitácion. En somme, le verbe performatif vouloir démontre l'accomplissement, c'est-à-dire la performance de l'acte Je vais rester plus content qu'est l'intention de l'apprenante. Si la philosophie du langage ordinaire soutient l'analyse sur ce verbe, c'est qu' « en le disant, on le fait » selon Vanderveken (p. 10) reprenant le propos austinien.

Or, une telle analyse démontrant la performance par le contexte énonciatif, démontre alors les raisons de l'évolution de la philosophie du langage ordinaire vers la philosophie contemporaine du langage. Si Searle \& Vanderveken (1985) reprennent en ce sens la théorie austinienne des actes de discours (1962), l'apprenante exprime alors l'intention d'être plus contente comme proposition avec et selon Vanderveken (1992, p. 12), «le but de représenter comment les choses sont dans le monde », ou mieux, l'attitude de l'apprenante lié à un état de choses associé aux sentiments d’une volonté pendant la sortie au cinéma. Malgré que la condition de satisfaction ne soit pas explicite dans le contexte énonciatif comme nous l'avons analysé précédemment, nous pouvons la déterminer par la condition de vérité sur le monde, lorsque ledit auteur signal que la notion de satisfaction est « une généralisation évidente de la notion de condition de vérité qui est nécessaire pour quantifier sur toutes les forces illocutoires » (p.15).

Si le champ de la philosophie analytique en logique sémantique extensionnelle peut définir la véridicité d'un état de choses qui rend vrai l'événement futur, le moment d'être accompagnée au cinéma, et par le mode subjonctif que tu m'accompagnes, le champ de la philosophie contemporaine du langage en théorie des actes de discours associe l'événement à un monde, c'est-à-dire au quantificateur au cinéma sur la force illocutoire d'assertion ou l'attitude d'assertion de l'apprenante donnée par la locution verbale vouloir t'inviter et envisagée par le verbe accompagner. Et ceci définit l'état de choses par le but illocutoire expressif 
de la proposition principale Je serais plus contente, à savoir, un tel état de choses se représente par un acte de sentiment et de volonté signifié par ses objets plus contente.

La reprise de Je écris à toi parce que je veux une invitácion est maintenant vérifiée : Je t'écris parce que je veux t'inviter est cohérent lorsque l'intention, la signification, la communication et l'action sur l'énoncé Je serais plus contente que tu m'accompagnes furent analysées. Bien sûr, l'hésitation sur l'emploi de la forme réfléchie du pronom toi dans je veux inviter toi découle déjà de l'emploi de sa forme disjointe dans la proposition antéposée Je écris à toi. L'apprenante n'a pas souhaité répéter, a fait preuve d'un manque de distinction entre ces deux formes et de connaissances quant à l'utilisation du pronom complément d'objet aussi bien direct te qu'indirect te. En revanche, l'apprenante a une attitude d'assertion aussi bien avant et après la reprise de son énoncé, lorsqu'appliquée par le verbe vouloir.

Dans l'optique de l'hypothèse de la forme logique, nous allons démontrer l'importance d'un tel outil permettant d'étendre l'analyse des deux significations serais et rester au contexte énonciatif. Le courant de la philosophie contemporaine du langage le permet, à l'inverse de celui de la philosophie du langage ordinaire. En effet, lorsqu'est entendu par « ordinaire » l'isolement de l'énoncé pour l'analyse de sa signification, nous sommes restreint à indifférencier la signification de l'objet être dans la proposition Je serais plus contente et rester dans Je vais rester plus content. Or, l'outil ou la formule résumée de Searle (1969), Force illocutoire F (Contenu propositionnel P), agit en comparant deux énoncés simples associés à une force illocutoire identifiée dans le contexte énonciatif, celle qui rend en somme plus pertinente la signification (le contenu propositionnel qu'est l'état de chose) respective des deux énoncés en comparaison. Comparons:

- F Vouloir/force illocutoire d'assertion (P Je vais rester plus contente);

- F Vouloir/force illocutoire d'assertion (P Je serais plus contente).

Signalons la citation de Vanderveken (1992, p. 11) : « Pour que deux énoncés élémentaires aient la même signification dans la langue, il faut que leurs énonciations littérales possibles dans les mêmes contextes d'emploi aient à la fois la même force illocutoire et le 
même contenu propositionnel ». Reprenons que serais signifie une intention circonstancielle, le temps d'être accompagné au cinéma, et que rester une intention illimitée. Déduisons la pertinence pour l'objet serais, puisque l'objet cinéma est le quantificateur ou un monde avec des séances audiovisuelles et ses horaires. Ainsi, le résultat de cette opposition propose une correction de l'erreur.

Enfin, soumettons cette pertinence à l'analyse de sa condition de vérité pour la pertinence de la condition de satisfaction recherchée : L'action future de l'apprenante, son comportement le temps d'être accompagnée au cinéma. Dans le courant de la philosophie analytique, le fondement théorique frégéen exige la juxtaposition d'une expression à d'autres termes considérés des dénotations. L'objectif d'une telle logique sémantique est l'extension, c'est-à-dire la satisfaction de l'expression en signifiant quelque chose et lorsque forme de l'expression et son contenu propositionnel sont en liaison pour l'analyse de la signification. Il y a en somme une convergence entre la condition de satisfaction et la condition de vérité : leur objectif est la signification. Ce que généralisent Searle \& Vanderveken (1985) est l'énoncé au contexte énonciatif quel que soit son type syntaxique, alors que Frege (1892) centre l'analyse des significations sur les énoncés déclaratifs d'énonciation assertive.

Réalisons l'exemple suivant pour identifier l'extension du concept cinéma, ainsi la pertinence de l'objet serais : Sous ce concept, les objets téléspectateur; affiche; horaire; salle, projecteur; lumière; son ont une propriété, séance audiovisuelle. L'extension consiste alors dans la propriété du concept cinéma. Et ce concept juxtaposé à ses objets constitue le contenu propositionnel, c'est-à-dire le monde (cinéma + ses objets) comme valeur de vérité sur la signification du concept cinéma. Et ce même monde, pour avoir comme propriété séance audiovisuelle, a un horaire ou un espace de temps limité qui produit alors la valeur de satisfaction sur la signification de serais. Enfin et encore plus pertinent lorsque lié à l'état de chose plus contente, le sentiment de volonté de l'apprenante réalise aussi l'action future, au delà de son intention, par un milieu audiovisuel animé par tous ses objets, et lesquelles rendent possible d'être accompagné par le destinataire du courriel de l'apprenante.

En somme, une telle analyse dans la mesure où les trois actes de parole inviter et répondre à une invitation, demander et indiquer l'beure et prendre et fixer un rendez-vous, 
contextualisant la tâche finale dans ladite méthode de français, présentent un mode de fonctionnement sémantique théorisé par des procédures langagières amenant l'apprenante à s'engager sur la vérité de ce qu'elle rédige et dit. Selon Vanderveken (1992, p. 10) «C’est lors de l'accomplissement de tels actes que les êtres humains expriment et communiquent leurs pensées ». La pensée ainsi formée dans la structure logique profonde du langage pour son expression dans la forme logique de l'énonciation réalise le sens, c'est-à-dire la signification, la communication, l'intention et l'action liées par le même contexte énonciatif. La performance est ainsi définis et comme Vanderveken nous croyons que « la compétence

linguistique ne peut pas être séparée de la performance, contrairement aux idées de Chomsky» (p.24). Nous provoquons une hypothèse: cette analyse sur la pensée comme sens (le tout signification/communication/intention/action) pour les énoncés du contexte énonciatif, contribuerait aux règles qui relationnent les structures syntaxiques avec les représentations de signifié.

\section{Considérations finales}

Pour la signification et l'action, la manière dont l'apprenante pourrait construire le verbe accompagner sur le plan syntaxique, associe cependant l'interprétation de l'action d'accompagner à une incertitude quant à la valeur de vérité du procès subordonné lorsque le subjonctif l'exprime. Nous avons alors vérifié le contexte énonciatif : Le laps de temps au cinéma comme point de l'événement positionne le procès subordonné dans l'avenir, par rapport au point de l'énonciation donné par le verbe accompagner. Le subjonctif est alors ici l'expression du futur, l'action à venir.

Ainsi, nous pouvons dire de la signification, c'est-à-dire de la valeur de vérité, qu'elle est lié à l'action, signification étant celle du verbe serais dans la proposition principale Je serais plus contente. Développer ce lien a permis d'expliquer la description des valeurs sémantiques (temporelle et aspectuelle) que ne réalisent pas les grammaires structurelles, mais aussi de tracer l'esquisse d'une règle absente dans la grammaire générative : cette règle relationnerait le mode subjonctif du verbe accompagner, c'est-à-dire la structure syntaxique de 
ce verbe, avec la dénotation du verbe principal serais, c'est-à-dire le laps de temps d'accompagner au cinéma dans l'avenir, ou mieux, la représentation du signifié serais.

Et sur le plan de la communication et de l'intention, ainsi, la liaison par la conjonction de subordination que de la proposition subordonnée complétive que tu m'accompagnes avec la proposition principale Je serais plus contente. Nous pensons que cette liaison ou que cette conjonction produit la communication en liant l'intention à l'action, ainsi à la signification. En effet, si cette classe grammaticale réalise la communication, notion citée quant à la critique de Roulet (1972) sur la grammaire générative, elle véhicule alors l'intention de l'apprenante en ce sens qu'elle n'est pas un simple complémenteur seulement responsable de la construction syntaxique du verbe, quant à son mode subjonctif et comme le voudrait ladite grammaire.

La réalisation de cette classe manifeste le sémantisme de l'énoncé complexe lorsque l'intention de l'apprenante est externe à la subordonnée qui la reçoit par accompagnes. Alors, la forme en étude, lorsque le mode conditionnel serais a la modalité d'incertitude et l'aspect co-verbal plus contente la modalité de volonté, nous donne la définition de l'intention prise dans la proposition principale. Si nous estimons que cette double intention, ou double modalité dans les termes du champ de l'énonciation, est reçue par le mode subjonctif que tu m'accompagnes, alors que transmet la communication entre l'ensemble des modalités dans les deux aspects (propositions simples ou modus et dictum selon les terme de l'énonciation). Ces participations conjointes pour le sens de l'ensemble seraient-elles la définition de la signification du tout?

\section{ANÁLISE DE UMA PRODUÇÃO ESCRITA E ORAL: SENTIDO EM CONTEXTO ENUNCIATIVO DO FRANCÊS LÍNGUA ESTRANGEIRA}

RESUMO: Afirmar hoje que o erro sintático e a confusão lexical sejam inexistentes nos enunciados de aprendizes do Français Langue Seconde (FLS), está erado. Não são, portanto, as contribuições teóricas da linguística estrutural e da psicologia behaviorista dos anos 1950 que corrigem de acordo com uma pedagogia de ensino ainda já com base na abordagem por competências de comunicação. Se compartilharmos por experiência algumas falhas no método de francês Latitudes 1 (2008) a ser aplicado pelo manual do professor, tudo se opõe a ensinar o modo de combinação junto com a interpretação dos objetos, a fim de aprender as estruturas sintáticas dos enunciados escritos e a interpretação delas na oralidade. E isso, apesar da frequência de atividades de repetição de expressões simples 
em contexto determinado pelos três atos de linguagem inviter et répondre à une invitation, demander et indiquer l'heure e, prendre et fixer un rendez-vous, os quais contextualizam uma tâche finale submetida à análise. Para entender melhor as regras que formam as estruturas sintáticas e as representações de significado nos enunciados dos aprendizes, parece relevante desenvolver pela abordagem analítica e contemporânea da filosofia da linguagem. Após uma parte dedicada à apresentação do corpus de referência e ao relatar a complexidade do problema de pesquisa, analisaremos uma produção escrita de aprendiz pelas ferramentas das referidas abordagens sobre o seguinte objeto de estudo: as descrições sintáticas e as representações de significado.

PALAVRAS-CHAVE: Mundo; Sentido; Significado; Sintática; Verbo.

\section{RÉFÉRENCES}

AUSTIN, John Langshaw. How to do things with words. Cambridge: Harvard University Press, 1962.

BÉRARD, Evelyne. L'approche communicative. Théorie et pratiques. Paris: CLE Internationale, 1991.

CANALE, Michael; SWAIN, Merrill. Theoretical Bases of Communicative Approaches to Second Language Teaching and Testing. Applied Linguistics, Oxford, vol. 1, n. 1, pp. 147, março 1980. Disponível em: < http://dx.doi.org/10.1093/applin/I.1.1 >. Acesso em: 12 maio 2019.

CHOMSKY, Noam. Linguagem e mente. Trad. Roberto Leal Ferreira. São Paulo: Editora UNESP, 2009.

DAVIDSON, Donald. Truth and Meaning. In: Synthese, 17, pp. 304-323, 1967.

GOTTLOB FREGE, Friedrich Ludwig. Begriffsschrift, eine der arithmetischen nachgebildete Formelsprache des reinen Denkens. Halle a. S., L. Nebert, 1879, X, 88 p.

. Über Sinn und Bedeutung. In: Zeitschnift für Philosophie und philosophische Kritik, Neue Folge, Band 100/1, Jg. 1892, S. 25-50.

KELLER, Philipp. Introduction à la logique. La logique classique des propositions et des prédicats. Genève, 2007. 357 p. Département de Philosophie, Université de Genève.

MÉRIEUX, Régine; LOISEAU, Yves. LATITUDES. 1: méthode de français. Paris : Didier, 2008.

MOIRAND, Sophie. Enseigner à communiquer en langue étrangère. Paris : Hachette, 1982.

ROULET, Eddy. Théories grammaticales, descriptions et enseignement des langues. Paris: F. Nathan, 1972.

SEARLE, John Rogers. Speech Acts: An Essay in the Philosophy of Language. Cambridge: Cambridge University Press, 1969. 
SEARLE, John Rogers.; VANDERVEKEN, Daniel. Foundations of Illocutionary Logic. Cambridge: Cambridge University Press, 1985.

VANDERVEKEN, Daniel. La théorie des actes de discours et l'analyse de la conversation. Cabiers de Linguistique Française, Genève, n. 13, pp. 8-61, 1992. Disponível em: $<$ https://clf.unige.ch/numeros/13/>. Acesso em: 25 maio 2019.

Recebido em: 13/08/2020.

Aprovado em: 15/10/2020. 